\title{
Uptake and bioavailability of anthocyanins and phenolic acids from grape/blueberry juice and smoothie in vitro and in vivo
}

\author{
Sabine Kuntz ${ }^{1 *}$, Silvia Rudloff ${ }^{1,2}$, Heike Asseburg ${ }^{1}$, Christian Borsch $^{1}$, Bettina Fröhling ${ }^{3}$, \\ Franziska Unger ${ }^{4}$, Sebastian Dold ${ }^{4}$, Bernhard Spengler ${ }^{4}$, Andreas Römpp ${ }^{4}$ and Clemens Kunz ${ }^{1}$ \\ ${ }^{1}$ Institute of Nutritional Science, Justus-Liebig-University Giessen, Wilhelmstrasse 20, 35392 Giessen, Germany \\ ${ }^{2}$ Department of Pediatrics, Justus-Liebig-University Giessen, 35392 Giessen, Germany \\ ${ }^{3}$ Geisenheim Research Center, Section of Wine Analysis and Beverage Technology, Geisenheim, Germany \\ ${ }^{4}$ Institute of Inorganic and Analytical Chemistry, Justus-Liebig-University, Giessen, Germany
}

(Submitted 1 August 2014 - Final revision received 10 December 2014 - Accepted 5 January 2015 - First published online 17 March 2015)

\begin{abstract}
The goal of eating five servings of fruits and vegetables a day has not yet been achieved. The intake of polyphenols such as anthocyanins (ACN) could be improved by consuming smoothies and juices that are increasingly popular, especially in children; however, bioavailability data concerning food matrix effects are scarce. Thus, we conducted a randomised, cross-over, bioavailability study ( $n 10)$ to determine the bioavailability of ACN and their metabolites from an ACN-rich grape/blueberry juice ( $841 \mathrm{mg} \mathrm{ACN} /$ litre) and smoothie (983 mg ACN/litre) in vivo, and the uptake of a corresponding grape/blueberry extract in vitro. After the intake of beverage (0.33 litres), plasma and fractionated urine samples were collected and analysed by ultra-performance liquid chromatography coupled to MS. The most abundant ACN found in plasma and urine were malvidin and peonidin as native ACN and as glucuronidated metabolites as well as 3,4-dihydroxybenzoic acid (3,4-DHB); minor ACN (delphinidin, cyanidin and petunidin) were only detected as native glycosides. Plasma pharmacokinetics and recoveries of urinary metabolites of ACN were not different for juice or smoothie intake; however, the phenolic acid 3,4-DHB was significantly better bioavailable from juice in comparison to smoothie. In vitro data with absorptive intestinal cells indicated that despite their weak chemical stability, ACN and 3,4-DHB could be detected at the basal side in their native forms. Whether smoothies as well as juices should be recommended to increase the intake of potentially health-promoting ACN and other polyphenols requires the consideration of other ingredients such as their relatively high sugar content.
\end{abstract}

Key words: Bioavailability: Grapes: Blueberries: Anthocyanins: In vivo studies: In vitro studies

Consumption of fruits and vegetables (FV) has been found to be associated with a decreased risk of diet-related diseases such as obesity, diabetes, CVD, CHD, stroke and some types of cancer ${ }^{(1-4)}$. In spite of this knowledge, guidelines and programmes to promote a healthy lifestyle, the compliance with higher FV intake is less promising ${ }^{(5)}$. In particular, FV intake in children is still far below the recommendations, e.g. less than five FV servings per $\mathrm{d}^{(6,7)}$. Therefore, the increase in FV intake in the form of juices or smoothies is very popular, especially in young age groups, and could thus be a source of health-promoting polyphenols with antioxidant and antiinflammatory activities ${ }^{(8)}$. Polyphenols such as anthocyanins $(\mathrm{ACN})$ are present in FV. High amounts of polyphenols are found in red and purple coloured fruits and account for $50-80 \%$ of the total polyphenol content in berries. Grapes, blueberries, blackberries, cherries or cranberries can reach concentrations of up to $3000 \mathrm{mg} / \mathrm{kg}$ fresh weight ${ }^{(9-11)}$. It is estimated that the average total intake of ACN may be approximately $200 \mathrm{mg} / \mathrm{d}$, which is four times higher than that of other polyphenols ${ }^{(12,13)}$. The main glycosylated ACN in fruits are cyanidin, delphinidin, malvidin, pelargonidin, peonidin and petunidin. Despite their high content, the bioavailability of ACN is considered to be relatively low compared with that of other flavonoids ${ }^{(14)}$. Studies investigating the pharmacokinetics of cyanidin or malvidin have shown that cyanidin-3-glucoside (Cya-3-glc) or malvidin-3-glucoside

Abbreviations: 3,4-DHB, 3,4-dihydroxybenzoic acid; ACN, anthocyanin; $C_{\max }$, maximal concentration; Cya-3-glc, cyanidin-3-glucoside; Del-3-glc, delphinidin-3-glucoside; DMEM, Dulbecco's modified Eagle's medium; FCS, fetal calf serum; FV, fruits and vegetables; Mal-3-glc, malvidin-3-glucoside; Mal-3-glucuro, malvidin-3-glucuronide; PA, phenolic acid; Peo-3-glc, peonidin-3-glucoside; Peo-3-glucuro, peonidin-3-glucuronide; Pet-3-glc, petunidin3-glucoside; SPE, solid-phase extraction; TEAC, Trolox equivalent antioxidant capacity; $t_{\text {max }}$, time to maximal concentration; UPLC-MS, ultraperformance liquid chromatography MS.

* Corresponding author: Dr S. Kuntz, fax + 49641 9939049, email sabine.kuntz@ernaehrung.uni-giessen.de 
(Mal-3-glc) reach a maximum plasma level at 30-60 min after ingestion with concomitant enrichment in urine after $2 \mathrm{~h}$. In contrast, cyanidin or malvidin glucuronide or methylated glucuronides were detected in urinary concentrations that were four to six times higher compared with native Cya-3-glc or Mal-3-glc ${ }^{(15,16)}$. Although in most studies glucuronidation or methylation seem to be the predominant phase II metabolisms in the liver, few studies have also identified sulphation of the native forms ${ }^{(17,18)}$. However, in all these studies, the bioavailability is less than $2 \%$ with concentrations of native $\mathrm{ACN}$ found in the plasma ranging from 1 to $100 \mathrm{nmol} / \mathrm{l}^{(19,20)}$. These findings of low concentrations in plasma and urine may be a consequence of the ACN instability at physiological $\mathrm{pH}$ and their degradation by the intestinal microflora ${ }^{(21-23)}$. While most studies have focused on absorption, metabolism and excretion of single or mixed ACN with controversial results, little information is available on the effects of the food matrix on their bioavailability ${ }^{(24)}$. Recently, it has been shown that the availability of ACN from berry juice is lower than that from berries ${ }^{(25,26)}$. In contrast, Hagl et al. ${ }^{(27)}$ demonstrated that polyphenols from an apple smoothie reached the colon, and their availability for the intestinal microbiota could be more effective in the prevention of chronic colon diseases than apple juice or apple cider. In addition, Nurmi et $a l .{ }^{(28)}$ investigated the occurrence of $\mathrm{ACN}$ and phenolic acids (PA) in urine after ingestion of fruit puree with and without oat cereals. They observed a delayed maximum urinary excretion of dietary PA after consumption of puree and cereal compared with consumption of puree alone ${ }^{(28)}$. We aimed at investigating whether the in vivo intake of ACNrich beverages such as grape/blueberry juice or smoothie, which are very popular in young age groups, differed with regard to the bioavailability of phenolic compounds. Furthermore, the in vivo bioavailability was compared with in vitro studies using extracts with a comparable ACN pattern.

\section{Materials and methods}

\section{Chemicals}

All chemicals were of analytical grade. Formic acid and ascorbic acid were from Sigma-Aldrich. Pelargonidin-3-Orutinoside was purchased from Extrasynthese; 2-chloro-5nitrobenzoic acid, formic acid and water (HPLC grade) from Fluka; trifluoroacetic acid and methanol from Merck; and Mal-3-glc from Roth.

\section{In vitro and in vivo study design}

\section{In vitro studies}

Cell culture. The human colon adenocarcinoma cell line Caco-2 (ATCC ${ }^{\mathcal{O}} \mathrm{HTB}_{3} 7^{\mathrm{TM}}$ ) was obtained from the American Type Culture Collection (LGC Standards GmbH). Cells were routinely grown in $75 \mathrm{~cm}^{2}$ culture flasks using Dulbecco's modified Eagle's medium (DMEM) at $\mathrm{pH} 7 \cdot 4$ with $1 \%$ non-essential amino acids and $20 \%$ fetal calf serum (FCS). Cells were maintained in a humidified atmosphere of $5 \% \mathrm{CO}_{2}$ at $37^{\circ} \mathrm{C}$. Stock passages were subcultured every $3-4 \mathrm{~d}$. For incubation studies, pre-confluent cells were trypsinised with a $0.25 \%(\mathrm{w} / \mathrm{v})$ trypsin/0.53 mm-EDTA solution, and $1 \times 10^{4}$ cells were seeded onto a transwell insert with a polycarbonate membrane (diameter $6.35 \mathrm{~mm}$, pore size $0.4 \mu \mathrm{m}$; Becton, Dickinson and Company) and placed in a twenty-four-well plate. Cells were allowed to grow to confluence and to differentiate to absorptive enterocytes within $21 \mathrm{~d}$. The culture medium was changed every $2-3 \mathrm{~d}$ at the apical $(0.35 \mathrm{ml})$ and basal side $(1.35 \mathrm{ml})$. Transepithelial electrical resistance was monitored using a Millicell-ERS Volt-Ohm Meter (Millipore). Only transwell filters with transepithelial electrical resistance values above $350 \Omega \mathrm{cm}^{2}$ were used for absorption experiments ( $n 3$ in duplicates).

Degradation and incubation studies. For incubation studies, inserts with cells were carefully washed at $37^{\circ} \mathrm{C}$ with DMEM containing $10 \%$ FCS. Apical compartments representing the luminal side of the gut and basal compartments representing the serosal side were filled for equilibration $\left(30 \mathrm{~min}, 37^{\circ} \mathrm{C}\right.$ ) with 0.5 and $1.5 \mathrm{ml}$ of medium (DMEM, $10 \%$ FCS), respectively. Then, grape/blueberry extract dissolved in medium was added to the apical compartment. The concentration of total ACN as the sum of individual ACN was 2613 (sEm 19) $\mu \mathrm{mol} / \mathrm{l}$, respectively 1290 (sEM 9) $\mathrm{mg} / \mathrm{l}$ (Table 2). ACN-rich extract was dissolved in cell culture medium (DMEM, $10 \% \mathrm{FCS}$ ) at $37^{\circ} \mathrm{C}$. The $\mathrm{pH}$ was adjusted to $7 \cdot 4$ for incubation studies and to $2 \cdot 0$ or $7 \cdot 4$ for degradation studies. The final concentration of the extract solution was about $50 \mu \mathrm{mol} / 1 \mathrm{Mal}-3$-glc equivalents. After 0, 30, 60 and $90 \mathrm{~min}$, medium from the apical and basal compartments was removed, mixed with $1 \%$ formic acid to prevent further degradation and stored immediately at $-80^{\circ} \mathrm{C}$ until ACN quantification. Control chambers without cells were run as a control to measure ACN degradation at $\mathrm{pH} 2 \cdot 0$ and $7 \cdot 4$. At the same time points, aliquots were taken and treated in the same way as the samples that had cell contact. ACN in apical compartments were analysed by HPLC with UV detection (HPLC-UV). Due to lower concentrations, basal compartments were analysed by ultra-performance liquid chromatography coupled to MS (UPLC-MS) after solid-phase extraction (see 'Analysis of in vitro and in vivo samples' section).

\section{In vivo studies}

Design and study subjects. From May to August 2010, we conducted a randomised, double-blind, cross-over study to investigate the bioavailability of ACN from ACN-rich juice and smoothie in healthy young individuals at the JustusLiebig University Giessen (Germany). The present study was carried out according to the guidelines laid down in the Declaration of Helsinki, and the local ethics committee (registration no. 13/10) approved all procedures involving human subjects. Written informed consent was obtained from all participants. Cluster sample size calculation for $2 \times 2$ cross-over designs with type 1 and 2 errors of 0.05 and $0 \cdot 2$, respectively, was performed with Stata version 12 (clustersampsi) (StataCorp). Power calculation estimated the required sample size to be ten (five per arm). Healthy subjects ( $n$ 10: five females and five males) between 23 and 27 years of age 
(25.0 (SEM 2.8) years) and with a BMI between 19.6 and $25.1 \mathrm{~kg} / \mathrm{m}^{2}\left(22.4(\operatorname{sem} 3.9) \mathrm{kg} / \mathrm{m}^{2}\right)$ were recruited. They were deemed eligible for the study in consideration of specific exclusion criteria ( 3 months without medications, vitamin and mineral supplementation). In a double-blind fashion, the included subjects were randomly assigned to the initial arm of the study for juice or smoothie. After randomisation, a 10-d washout period was followed by the intervention day. After an overnight fast, the participants ingested 0.33 litres of juice or smoothie; blood $(0,10,20,30,60,90$ and $120 \mathrm{~min})$ and urine (fractions of $0-3$, 3-6, 6-9, 9-12, 12-15 and 15-24h) samples were taken. Participants were allowed to consume only water within $3 \mathrm{~h}$ after ingestion of the study juice or smoothie. A $14 \mathrm{~d}$ run-out finished the first cycle. This procedure was performed twice for juice or smoothie. The present study design is shown in Fig. 1.

Source of anthocyanin-rich juice, smoothie and extract. Juice, smoothie and extract were produced at the Geisenheim University (Department of Wine Chemistry and Beverage Technology, Research Center Geisenheim) and made from an eighty/twenty mixture of red grapes (grape varieties Dakapo and Accent) and blueberries (Vaccinium myrtillus) Briefly, grapes were extruded, and the resulting juice was separated, blended with blueberry juice from concentrate, pasteurised and hot-filled into 0.33 litres of brown glass bottles. Smoothie was produced from juice being blended with $20 \%$ blueberry puree instead of blueberry juice. Juice was analysed directly after membrane filtration (pore size $0.45 \mu \mathrm{m}$ ) for basic analytical parameters such as total phenolics, concentrations of ACN and Trolox equivalent antioxidant capacity (TEAC) ${ }^{(29,30)}$. For HPLC and TEAC analyses, smoothie was extracted twice with $80 \%$ methanol. Corresponding extract for in vitro studies was made from juice. Briefly, grape/blueberry juice was loaded onto a pilot glass column (Kronlab/Schott, $200 \times 750 \mathrm{~mm}$ ) filled with adsorber resin (Resindion/Mitsubishi). Water-soluble juice constituents such as sugars, organic acids and minerals were washed out with two bed volumes of distilled water. The first eluate was slightly coloured due to low ACN levels. ACN and colourless polyphenols were eluted with two bed volumes of $96 \%$ ethanol. The ethanolic fraction was concentrated using a rotary evaporator and lyophilised.
Analysis of ACN as well as macro- and micro-nutrient content in juice and smoothie is described elsewhere ${ }^{(31)}$. 3,4-Dihydroxybenzoic acid (3,4-DHB) was analysed by UPLC-MS (see 'Analysis of in vitro and in vivo samples' section). The concentrations of ACN and 3,4-DHB are listed in Table 1.

After an overnight fast, volunteers consumed 0.33 litres of juice or smoothie. Volunteers ate a standardised lunch $5 \mathrm{~h}$ after drinking the beverage and thereafter remained on a low-polyphenol diet for a further $20 \mathrm{~h}$ until the final urine samples were collected. Venous blood samples $(30 \mathrm{ml})$ were collected in EDTA tubes from all volunteers at 0, 10, 20, 30, 40, 60, 90 and 120 min post-ingestion, and plasma was separated by centrifugation at $3000 \mathrm{~g}$ for $10 \mathrm{~min}$ at $4^{\circ} \mathrm{C}$. Plasma was divided into $1 \mathrm{ml}$ aliquots, each of which was acidified using $30 \mu \mathrm{l}$ of $50 \%$ aqueous formic acid and stored at $-80^{\circ} \mathrm{C}$ until further analysis. Urine was collected at baseline and over six time periods, $0-3,3-6,6-9,9-12,12-15$ and $15-24 \mathrm{~h}$, after ingestion of the test drink. The total volume for each period was recorded, acidified with $1 \%$ formic acid and aliquots were stored at $-80^{\circ} \mathrm{C}$ until further analysis.

\section{Analysis of in vitro and in vivo samples}

\section{Sample extraction}

Solid-phase extraction (SPE) was used for basal cell compartment samples as well as for plasma and urine samples due to lower concentrations of ACNs and 3,4 DHB. Before SPE, pelargonidin-3-O-rutinoside and 2-chloro-5-nitrobenzoic acid were added to the samples as internal standards. Plasma samples were extracted using a slightly modified method developed by Esselen et al. ${ }^{(32)}$. Briefly, SPE Waters Oasis ${ }^{\circledR}$ HLB (Waters) cartridges were conditioned with $1 \mathrm{ml}$ methanol and $1 \mathrm{ml} 1.5 \mathrm{M}$-formic acid before $1 \mathrm{ml}$ plasma sample was loaded onto the column. The column was then washed with $1 \mathrm{ml}$ of $1.5 \mathrm{M}$-formic acid and $1 \mathrm{ml}$ methanol (5\%). Retained ACN were eluted with $1 \mathrm{ml}$ of $0 \cdot 1 \%$ formic acid in methanol. The eluate was reduced to dryness under a stream of $\mathrm{N}$ at $20^{\circ} \mathrm{C}$. Plasma preparations were reconstituted with $100 \mu \mathrm{l}$ of trifluoroacetic acid (5\% v:v) in water. Reconstituted samples were stored at $-80^{\circ} \mathrm{C}$ until further analysis. Urine and basal

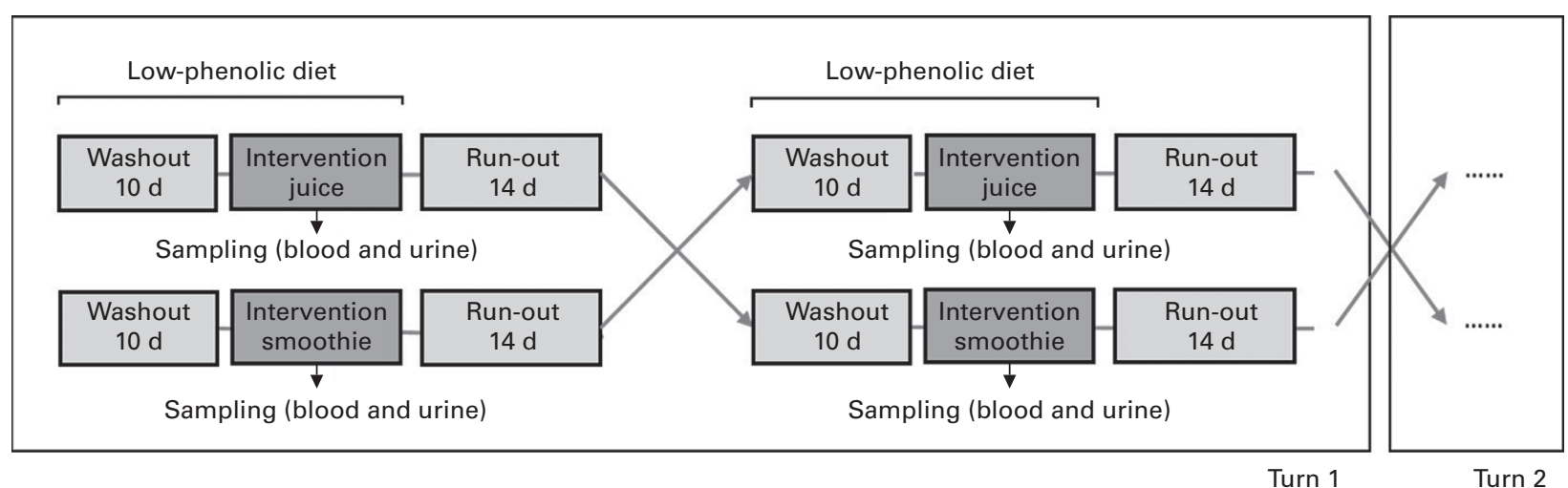

Fig. 1. Design of the bioavailability study. Study participants were randomly assigned to consume the test beverages. They passed two cycles getting juice and smoothie in a double-blind, cross-over design. One cycle was intermitted by a $10 \mathrm{~d}$ washout phase and a $14 \mathrm{~d}$ run-out phase. At the intervention day, the volunteers consumed the beverages after an overnight fast. Blood and urine samples were taken as described in the 'Materials and methods' section. All the subjects completed the study $(n 10)$. 
Table 1. Anthocyanins (ACN) and 3,4-dihydroxybenzoic acid (3,4-DHB) in beverages (ACN-rich juice and smoothie) and the corresponding extract ${ }^{\star}$ (Mean values and standard deviations, $n 2$ )

\begin{tabular}{|c|c|c|c|c|c|c|c|c|c|c|c|c|}
\hline \multirow[b]{2}{*}{ Phenolic } & \multicolumn{2}{|c|}{ Juice (mg/l) } & \multicolumn{2}{|c|}{$\begin{array}{c}\text { Juice } \\
(\mu \mathrm{mol} / \mathrm{l})\end{array}$} & \multicolumn{2}{|c|}{$\begin{array}{l}\text { Smoothie } \\
(\mathrm{mg} / \mathrm{l})\end{array}$} & \multicolumn{2}{|c|}{$\begin{array}{l}\text { Smoothie } \\
(\mu \mathrm{mol} / \mathrm{l})\end{array}$} & \multicolumn{2}{|c|}{$\begin{array}{l}\text { Extract } \\
(\mathrm{mg} / \mathrm{l})\end{array}$} & \multicolumn{2}{|c|}{$\begin{array}{l}\text { Extract } \\
(\mu \mathrm{mol} / \mathrm{l})\end{array}$} \\
\hline & Mean & SD & Mean & SD & Mean & SD & Mean & SD & Mean & SD & Mean & SD \\
\hline 3,4-DHB & 11.5 & 0.72 & 71.8 & 4.8 & $9 \cdot 26$ & 0.15 & $60 \cdot 1$ & 0.4 & 0.94 & 0.09 & $6 \cdot 16$ & 0.68 \\
\hline Malvidin-3-glucoside & 273 & 4 & 554 & 8 & 274 & 10 & 556 & 20 & 419 & 2 & 849 & 3 \\
\hline Malvidin-3-(6"-p-coumaroyl)-glucoside & $66 \cdot 4$ & 0.3 & 104 & 1 & 46.5 & 1.9 & $72 \cdot 7$ & $3 \cdot 0$ & 103 & 1 & 161 & 1 \\
\hline Malvidin-3-(6"-O-acetyl)-glucoside & 51.4 & 0.7 & 96.6 & $1 \cdot 3$ & $46 \cdot 4$ & $2 \cdot 1$ & $86 \cdot 7$ & 3.9 & $87 \cdot 8$ & 0.5 & 164 & 1 \\
\hline Malvidin-3-arabinoside & 4.22 & 0.21 & $9 \cdot 1$ & 0.4 & 5.58 & $0 \cdot 12$ & 11.9 & 0.2 & 5.49 & 0.27 & 11.7 & 0.4 \\
\hline$\Sigma$ Malvidin & 396 & 5 & 764 & 11 & 373 & 14 & 728 & 27 & 615 & 3 & 1185 & 5 \\
\hline Peonidin-3-glucoside & 165 & 2 & 356 & 4 & 163 & 6 & 352 & 13 & 248 & 1 & 537 & 1 \\
\hline Peonidin-3-(6"-O-acetyl)-glucoside & $18 \cdot 3$ & 0.1 & $36 \cdot 2$ & 0.2 & 14.7 & $1 \cdot 2$ & $29 \cdot 1$ & $2 \cdot 4$ & $31 \cdot 2$ & 0.0 & 61.7 & 0.1 \\
\hline Peonidin-3,5-diglucoside & 5.94 & 0.45 & $9 \cdot 4$ & 0.6 & 8.04 & $0 \cdot 18$ & $12 \cdot 8$ & 0.2 & $7 \cdot 48$ & 0.82 & 11.8 & 1.3 \\
\hline Peonidin-3-galactoside & \multicolumn{2}{|c|}{ ND } & \multicolumn{2}{|c|}{ ND } & 4.40 & 0.06 & 9.54 & 0.08 & \multicolumn{2}{|c|}{ ND } & \multicolumn{2}{|c|}{ ND } \\
\hline$\Sigma$ Peonidin & 189 & 2 & 402 & 5 & 190 & 7 & 403 & 15 & 287 & 1 & 610 & 2 \\
\hline Delphinidin-3-glucoside & $67 \cdot 4$ & 1.1 & 144 & 2 & 105 & 3 & 225 & 7 & $96 \cdot 4$ & 1.3 & 207 & 3 \\
\hline Delphinidin-3-arabinoside & $10 \cdot 9$ & 0.1 & $25 \cdot 0$ & 0.2 & $30 \cdot 9$ & $1 \cdot 1$ & $71 \cdot 0$ & $2 \cdot 5$ & $14 \cdot 2$ & 1.0 & $32 \cdot 6$ & $2 \cdot 3$ \\
\hline Delphinidin-3-galactoside & $9 \cdot 37$ & 0.16 & $20 \cdot 0$ & 0.2 & 43.1 & 1.9 & $92 \cdot 6$ & $4 \cdot 1$ & $15 \cdot 5$ & 0.2 & 33.3 & 0.4 \\
\hline Delphinidin-3-(6"-O-acetyl)-glucoside & $5 \cdot 15$ & 0.26 & $10 \cdot 1$ & 0.4 & 6.59 & 0.64 & $12 \cdot 8$ & $1 \cdot 2$ & $9 \cdot 16$ & 0.32 & 17.9 & 0.6 \\
\hline$\Sigma$ Delphinidin & $92 \cdot 7$ & 1.5 & 199 & 3 & 185 & 7 & 402 & 15 & 135 & 3 & 291 & 6 \\
\hline Petunidin-3-glucoside & 69.8 & 1.4 & 145 & 2 & $87 \cdot 0$ & $3 \cdot 3$ & 181 & 6 & 103 & 1 & 216 & 2 \\
\hline Petunidin-3-galactoside & \multicolumn{2}{|c|}{ ND } & \multicolumn{2}{|c|}{ ND } & 11.9 & 0.5 & $24 \cdot 8$ & 1.0 & \multicolumn{2}{|c|}{ ND } & \multicolumn{2}{|c|}{ ND } \\
\hline Petunidin-3-(6"-p-coumaroyl)-glucoside & $15 \cdot 3$ & 0.1 & 24.5 & 0.2 & $12 \cdot 7$ & 0.8 & $20 \cdot 3$ & $1 \cdot 3$ & $29 \cdot 9$ & 0.2 & $47 \cdot 8$ & 0.3 \\
\hline Petunidin-3-(6"-O-acetyl)-glucoside & $10 \cdot 9$ & 0.1 & 20.9 & 0.6 & 8.44 & $1 \cdot 36$ & $16 \cdot 1$ & $2 \cdot 5$ & $17 \cdot 0$ & 0.1 & $32 \cdot 6$ & 0.2 \\
\hline$\sum$ Petunidin & 96 & 1 & 191 & 3 & 120 & 5 & 242 & 11 & 150 & 1 & 296 & 2 \\
\hline Cyanidin-3-glucoside & $41 \cdot 1$ & 0.5 & 91.4 & 1.4 & 58.4 & $2 \cdot 1$ & 129 & 5 & $62 \cdot 9$ & 0.5 & 140 & 1 \\
\hline Cyanidin-3-galactoside & $13 \cdot 4$ & 0.1 & 29.8 & 0.2 & $30 \cdot 2$ & 0.9 & $67 \cdot 2$ & $2 \cdot 0$ & $21 \cdot 8$ & 0.6 & 48.5 & 1.3 \\
\hline Cyanidin-3-arabinoside & $12 \cdot 0$ & 0.2 & 28.6 & 0.5 & $18 \cdot 6$ & 0.6 & $44 \cdot 3$ & 1.4 & $17 \cdot 4$ & 0.0 & 41.5 & 0.0 \\
\hline Cyanidin-3,5-diglucoside & \multicolumn{2}{|c|}{ ND } & \multicolumn{2}{|c|}{ ND } & 4.97 & 0.11 & 8.07 & 0.23 & \multicolumn{2}{|c|}{ ND } & \multicolumn{2}{|c|}{ ND } \\
\hline Cyanidin-3-(6"-O-acetyl)-glucoside & \multicolumn{2}{|c|}{ ND } & \multicolumn{2}{|c|}{ ND } & 2.55 & 0.59 & $5 \cdot 14$ & 1.03 & \multicolumn{2}{|c|}{ ND } & \multicolumn{2}{|c|}{ ND } \\
\hline$\Sigma$ Cyanidin & 66.5 & 0.8 & 149 & 1 & 114 & 4 & 254 & 9 & 102 & 1 & 230 & 2 \\
\hline Sum ACN & 840 & 11 & 1707 & 23 & 983 & 38 & 2031 & 79 & 1290 & 9 & 2613 & 18 \\
\hline
\end{tabular}

ND, not detectable.

*Beverages were analysed by HPLC-photodiode array/electrospray ionisation-MS or ultra-performance liquid chromatography-MS; for more details, see 'Materials and methods' section.

cell culture samples were performed according to Urpi-Sarda et al ${ }^{(33)}$ with slight modifications. Briefly, samples were centrifuged at $1500 \mathrm{~g}$ for $5 \mathrm{~min}$ at $20^{\circ} \mathrm{C}$. Samples $(1 \mathrm{ml})$ were loaded onto a preconditioned SPE Waters Oasis ${ }^{\circledR}$ HLB Cartridge (Waters $\mathrm{GmbH}$ ). Metabolites were eluted with $1 \mathrm{ml}$ acidified methanol after washing the column with $1 \mathrm{ml}$ of $1.5 \mathrm{M}$-formic acid and $1 \mathrm{ml}$ of $5 \%$ methanol. The eluate was reduced to dryness under a stream of $\mathrm{N}$ at $20^{\circ} \mathrm{C}$. Urine and basal cell culture samples were reconstituted with $100 \mu \mathrm{l}$ formic acid $(5 \% \mathrm{v}: \mathrm{v})$ and methanol (10\%) in water. Again, reconstituted samples were stored at $-80^{\circ} \mathrm{C}$ until further analysis.

\section{Identification and quantification of anthocyanins and 3,4-dihydroxybenzoic acid}

Apical cell culture samples were analysed by HPLC-UV. Due to lower concentrations, basal cell culture compartments, urine and plasma samples were analysed by UPLC-MS. Calibration curves in both cases were generated by using a grape extract with a known amount of Mal-3-glc and 3,4-DHB, which was used for the quantification of all ACN and 3,4-DHB.

HPLC-UV. A capillary HPLC system (Summit ${ }^{\circledR}$ Dionex $\mathrm{GmbH}$ ) equipped with a pump (P580 A HPG), an autosampler (ASI-100T Automated Sample Injector) and a reversed-phase column (type: inner diameter $6.35 \mathrm{~mm}$, pore size $0.4 \mu \mathrm{m}$, NUCLEOSIL 300 C18; Macherey-Nagel GmbH + Company $\mathrm{KG}$ ) as well as an UVD 340U (Dionex $\mathrm{GmbH}$ ) were used for analysis of apical cell culture compartments. Detection was carried out at a wavelength of $520 \mathrm{~nm}$. Mobile phases consisted of $5 \% \mathrm{v}: \mathrm{v}$ formic acid in water (solvent $\mathrm{A}$ ) and methanol (solvent B). The solvent gradient was $5 \% \mathrm{~B}$ at $0-2 \mathrm{~min}, 20 \% \mathrm{~B}$ at $10-15 \mathrm{~min}, 30 \% \mathrm{~B}$ at $30 \mathrm{~min}, 37 \% \mathrm{~B}$ at $35-40 \mathrm{~min}, 40 \% \mathrm{~B}$ at $41-43 \mathrm{~min}, 95 \% \mathrm{~B}$ at $50-62 \mathrm{~min}$ and $5 \% \mathrm{~B}$ at $70-80 \mathrm{~min}$ at a flow rate of $70 \mu \mathrm{l} / \mathrm{min}$. Injection volume was $15 \mu \mathrm{l}$.

UPLC-MS. UPLC separation was performed using an UltiMate 3000 Rapid Separation LC system (Dionex, Thermo Fisher Scientific Germany BV \& Co KG) equipped with a pump (HPG $3200 \mathrm{RS}$ ), an autosampler (WPS-3000 TRS) and a reversed phase (C18) column (type: $100 \mathrm{~mm} \times 2.1 \mathrm{~mm}$, particle size $2.6 \mu \mathrm{m}$, Kinetex; Phenomenex). Mobile phases consisted of $0.7 \% \mathrm{v}: \mathrm{V}$ formic acid in water (solvent A) and methanol (solvent B). The injection volume was $40 \mu \mathrm{l}$, and the solvent gradient was $2 \% \mathrm{~B}$ at $0-0.5 \mathrm{~min}, 7 \% \mathrm{~B}$ at $1 \mathrm{~min}$, $15 \% \mathrm{~B}$ at $5 \mathrm{~min}, 40 \% \mathrm{~B}$ at $10 \mathrm{~min}, 70 \% \mathrm{~B}$ at $13-16 \mathrm{~min}$ at a flow rate of $400 \mu \mathrm{l} / \mathrm{min}(p(\max )=550$ bar $)$. UPLC system was coupled to an Exactive Orbitrap Mass Spectrometer (Thermo Fisher Scientific) by an electrospray ion source. Capillary temperature was set to $350^{\circ} \mathrm{C}$, and spray voltage was $4.4 \mathrm{kV}$ in positive ion mode and $3.5 \mathrm{kV}$ in negative ion 
mode. Measurements were performed by continuously switching between positive and negative ion mode. Mass spectra were acquired in the range of $m / z 200-850$ for positive ion mode and $m / z 100-850$ for negative ion mode.

\section{Statistical and pharmacokinetic analyses of metabolites in} cell culture, plasma and urine samples

\section{In vitro analysis}

All data are presented as mean values with their standard errors ( $n 3$ in duplicates). Significant differences between the means of ACN degradation and incubation in transport chambers and the respective control chambers were assessed by one-way ANOVA. The significance of differences for ACN degradation and incubation was assessed using $t$ test. Differences were considered to be significant at $P<0.05$ and $P<0.01$.

\section{In vivo analysis}

Data on ACN and their metabolites are presented as mean values with their standard errors ( $n 10$ in duplicates). The Kolmogorov-Smirnov test was used to test the data for normal distribution, and significant differences between juice and smoothie were analysed by one-way ANOVA or paired $t$ test. Differences were considered to be significant at $P<0.05$.

The statistical IBM software SPSS for Windows (version 19.0.0; IBM Deutschland GmbH) and GraphPad Prism (a)
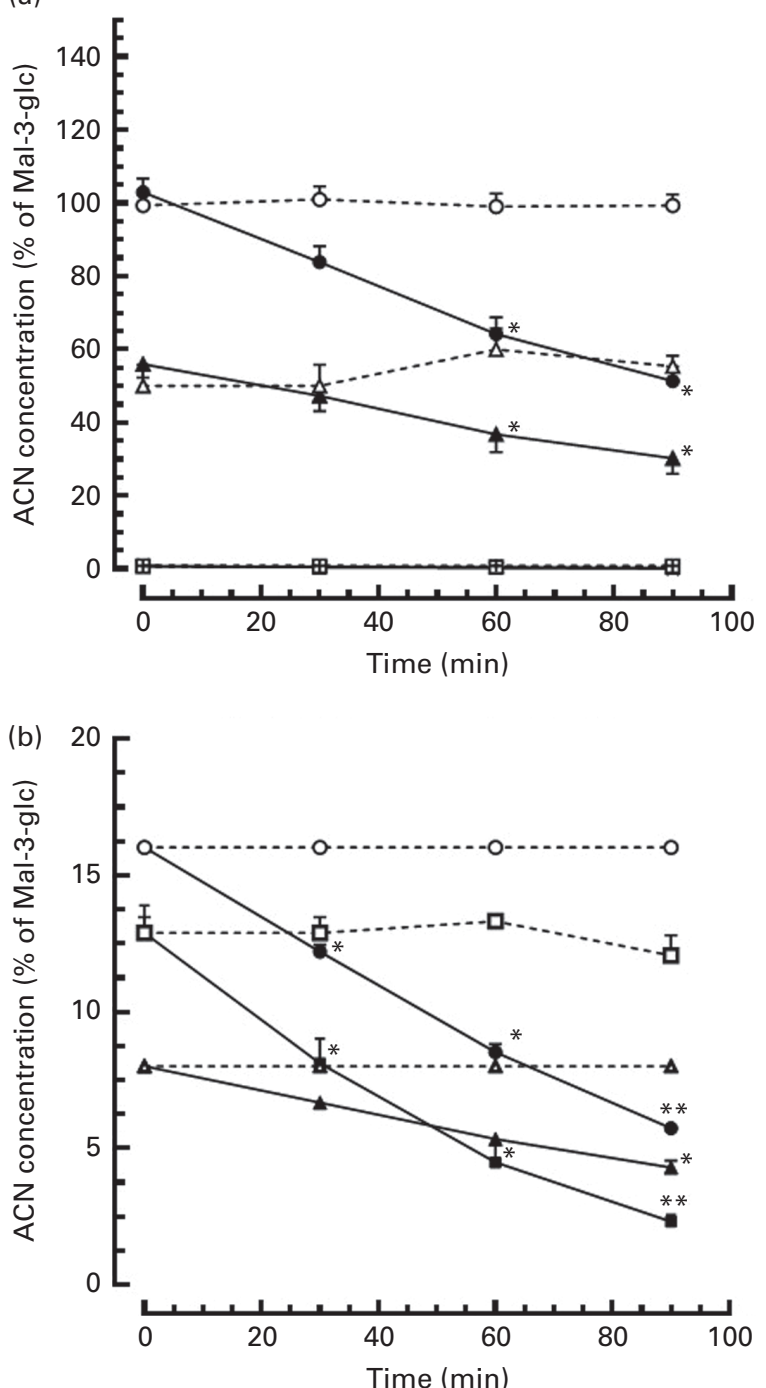

(c)
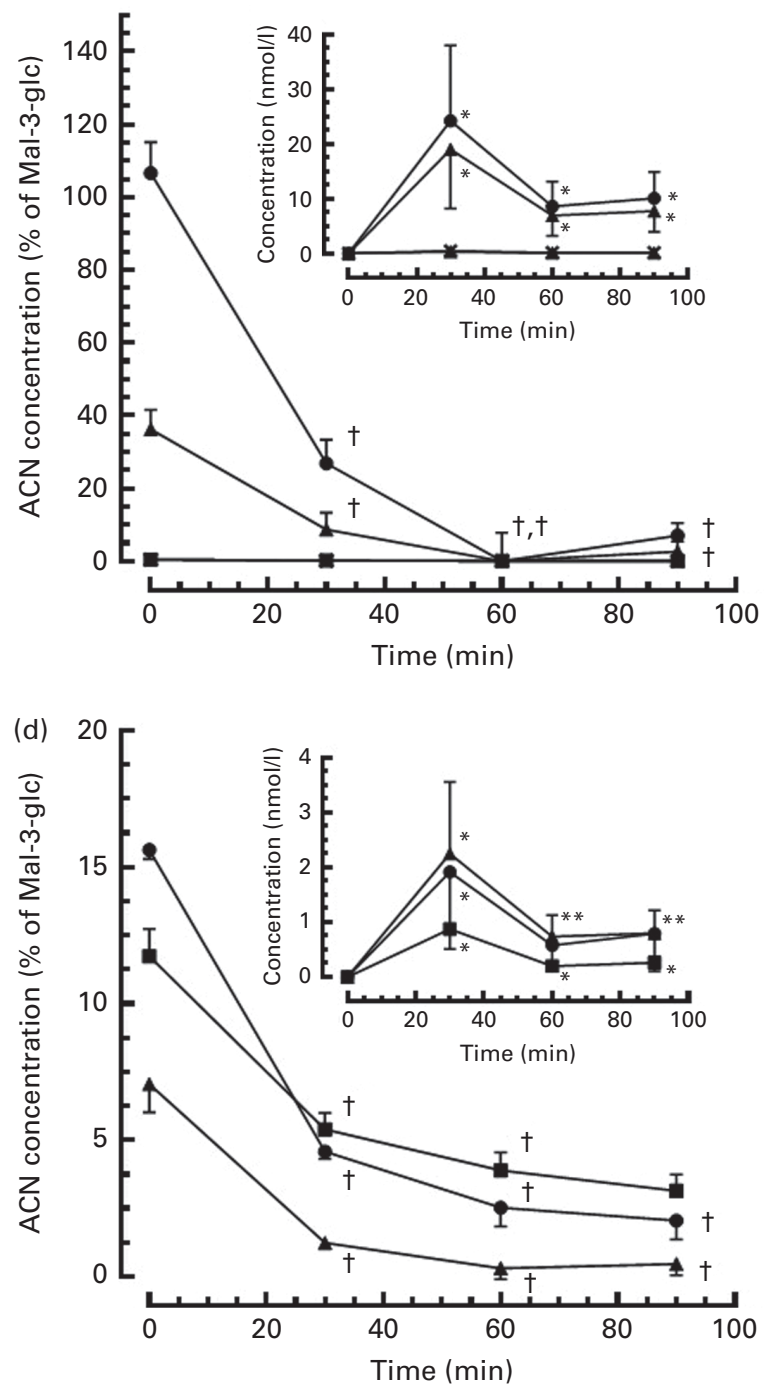

Fig. 2. Individual anthocyanin ( $\mathrm{ACN}$, in percentage of initial malvidin-3-glucoside (Mal-3-glc) concentration (50 $\mu \mathrm{M}$ Mal-3-glc equivalents)) over time in the apical compartments during degradation $(\mathrm{a}$ and $\mathrm{b})$ at $\mathrm{pH} 2.0(\ldots)$ and $\mathrm{pH} 7.4(-)$ and incubation (c and d) experiments ( $\mathrm{pH} 7.4)$. Basal appearance (nmol/l) of individual ACN is shown in insets. Values are means ( $n 3$ experiments each run in duplicates), with their standard errors represented by vertical bars. Mean value was significantly different to the initial concentrations: ${ }^{*} P<0.05,{ }^{* *} P<0.01$. † Mean value was significantly different between incubation (with cells) and degradation (without cells) chambers $(P<0.05)$. (a) - - , Mal-3-glc $(7.4) ;-\_$, peonidin-3-glucoside (Peo-3-glc $(7 \cdot 4)$ ); - - , malvidin-3,5-diglucoside (Mal-3,5-dglc $(7 \cdot 4)$ ); $\rightarrow-$, peo-

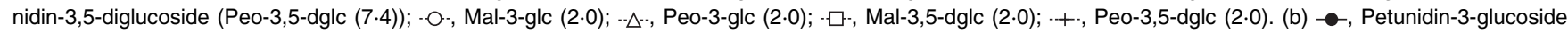

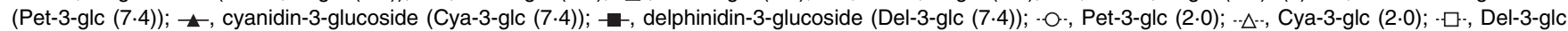

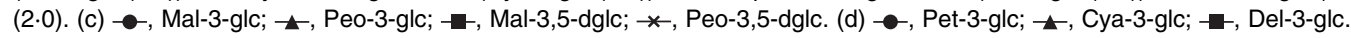


(version 6.0.2; GraphPad Software, Inc.) were used for pharmacokinetic data analysis.

\section{Results}

In vitro study

After application of the grape/blueberry extract to the apical compartments of transwell chambers, ACN (Mal-3-glc, peonidin-3-glucoside (Peo-3-glc), petunidin-3-glucoside (Pet-3glc), delphinidin-3-glucoside (Del-3-glc) and Cya-3-glc) degradation at $\mathrm{pH} 2 \cdot 0$ and $7 \cdot 4$ (without cells) was determined by HPLC-UV. Cellular transport of ACN from the apical to the basal compartment at $\mathrm{pH} 7 \cdot 4$ was determined by UPLC-MS. As shown in Fig. 2(a) and (b), all ACN concentrations decreased significantly over time in the degradation chambers (without cells) at $\mathrm{pH} 7 \cdot 4$, but not at $\mathrm{pH} 2 \cdot 0$, thus reflecting the stability at lower $\mathrm{pH}$. Significant differences between incubation media ( $\mathrm{pH} 7$ 4, with cells; Fig. 2(c) and (d) and the respective degradation media ( $\mathrm{pH} 7 \cdot 4$, without cells) became significant after $30 \mathrm{~min}$; correspondent enrichment in the basal compartment was observed for both native ACN, Mal-3-glc and Peo-3-glc, reaching a maximum after $30 \mathrm{~min}$ of incubation time (insets in Fig. 2(c) and (d)). Despite the higher concentration of Mal-3-glc in the extract compared with Peo-3-glc (50 and $31 \mu \mathrm{mol} / 1$, respectively), basal concentrations after $30 \mathrm{~min}$ were in the same range (24.5 (SEM 12.4) and 19.6 (SEM 13.4) nmol/1, respectively) revealing absorption rates of $0.05 \%$ for Mal-3-glc and $0.06 \%$ for Peo-3-glc. In contrast to the monoglucosylated ACN, the diglucosylated Mal-3,5-dglc and Peo-3,5-dglc were not transported in quantifiable concentrations (Fig. 2(a), inset). Regarding the minor ACN, petunidin (Pet-3-glc) and delphinidin (Del-3-glc) showed higher instability than cyanidin (Cya-3-glc) over time. Significant differences in the disappearance of Pet-3-glc, Del-3-glc and Cya-3-glc between degradation (without cells) and incubation (with cells) chamber were observed at $30 \mathrm{~min}$. Thus, absorption efficiencies appeared to be influenced by the structural skeleton since basal appearance of Cya-3-glc was much higher than that of Pet-3-glc or Del-3-glc (2.5 (SEM 1.3) v. 1.9 (SEM 1.1) and $0.8(\operatorname{sem} 0.4) \mathrm{nmol} / \mathrm{l})$ corresponding to recovery rates of $0.03,0.01$ and $0.005 \%$ of initial apical concentration for Cya-3-glc, Pet-3-glc and Del-3-glc, respectively.

For 3,4-DHB, no degradation was observed in the apical compartment of the transwell chambers (Fig. 3); however, cellular transport from the apical to the basal compartment was observed after $30 \mathrm{~min}$ of incubation time 5.5 (SEM $2 \cdot 4) \mathrm{nmol} / \mathrm{l} ; 2 \cdot 5 \%$ of the apical concentration).

\section{In vivo study}

The total amount of ACN in 0.33 litres juice and smoothie was 277.4 (SEM 3.8) and 324.5 (SEM 12.8) $\mathrm{mg}$ with the major ACN Mal-3-glc (90.2 (SEM 1.3) and 90.6 (sEm 3.3) mg) and Peo-3glc $(54.5$ (SEM 0.7$)$ and $53.8(\operatorname{sem} 2.0) \mathrm{mg}$ ) and the minor ACN Del-3-glc (22.2 (sem 0.4) and 34.6 (sem 1.1) mg), Cya-3glc (13.6 (sem 0.2) and 19.1 (sem 0.7) mg) and Pet-3-glc (22.8 (sem 0.5) and 28.7 (sem 1.1) mg) (Table 1). These five

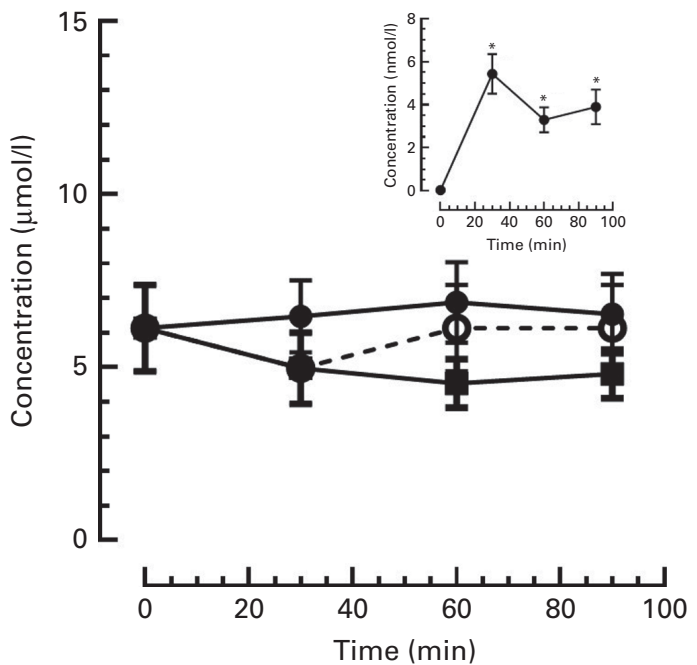

Fig. 3. 3,4-Dihydroxybenzoic acid (3,4-DHB) ( $\mu \mathrm{mol} / \mathrm{l})$ over time in apical compartments during degradation at $\mathrm{pH} 2.0$ (.....) and $\mathrm{pH} 7.4$ (-) and incubation experiments $(\mathrm{pH} 7.4)$; basal appearance $(\mathrm{nmol} / \mathrm{l})$ of $3,4-\mathrm{DHB}$ is shown in the inset. Values are means (n 3 experiments each run in duplicates), with their standard errors represented by vertical bars. * Mean value was significantly different to the initial concentrations $(P<0.05)$. - - , 3,4-DHB (pH 7.4, degradation); -0-, 3,4-DHB (pH 2.0, degradation); - 3,4-DHB (pH 7.4, incubation).

ACN accounted for about $80 \%$ of the ACN ingested via juice or smoothie, and were detected in plasma as well as in urine samples after consumption of the beverage by young and healthy volunteers (Fig. 4). ACN in baseline plasma and urine samples were absent before the ingestion of juice and smoothie, demonstrating the compliance of our subjects with dietary rules within the washout period. The occurrence of the five ACN named above in plasma reflected their concentration in juice and smoothie with Del-3-glc, Cya-3-glc and Pet-3-glc (Fig. 4(a)-(c)) as minor and Mal-3-glc and Peo-3-glc (Fig. 4(d) and (e)) as major compounds. UPLC-MS analysis of plasma samples not only revealed the occurrence of ACN glucosides in all subjects ( $n 10$ in duplicate), but also glucuronidated (glucuro) metabolites of malvidin and peonidin (Fig. 4(d) and (e)), although with wide individual variations. In addition to glucuronides, neither further phase II metabolites nor degradation products were found in plasma and urine.

Pharmacokinetic profiles of plasma ACN glucosides and glucuronides are shown in Fig. 4 and summarised in Table 2. After ACN intake, Mal-3-glc and Peo-3-glc were detected in plasma with maximal concentration $\left(C_{\max }\right)$ of 1.54 (SEm 0.66 ) and $0.80(\operatorname{sem} 0.32) \mathrm{nmol} / \mathrm{l}$ for juice and 1.00 (SEM 0.14) and 0.46 ( SEM 0.07) nmol/1 for smoothie, respectively. Both ACN had a similar time to maximal concentration $\left(t_{\max }\right)$ after juice or smoothie intake with a slightly delayed peak after smoothie ingestion (68 (SЕM 8) v. 75 (SEM 8) and 55 (SEM 3) v. 69 (SEM 6) $\mathrm{min}$ ).

Although the AUC for Mal-3-glc and Peo-3-glc revealed higher values after juice (103.1 (SEM 34.6) and 52.5 (SEM 16.6) $\mathrm{nmol} \times \mathrm{min} / \mathrm{l})$ than after smoothie (81.5 (SEM 11.4) and $37 \cdot 4(\operatorname{sem} 5 \cdot 5) \mathrm{nmol} \times \mathrm{min} / \mathrm{l})$ ingestion, there were no significant differences with regard to the bioavailability of these 

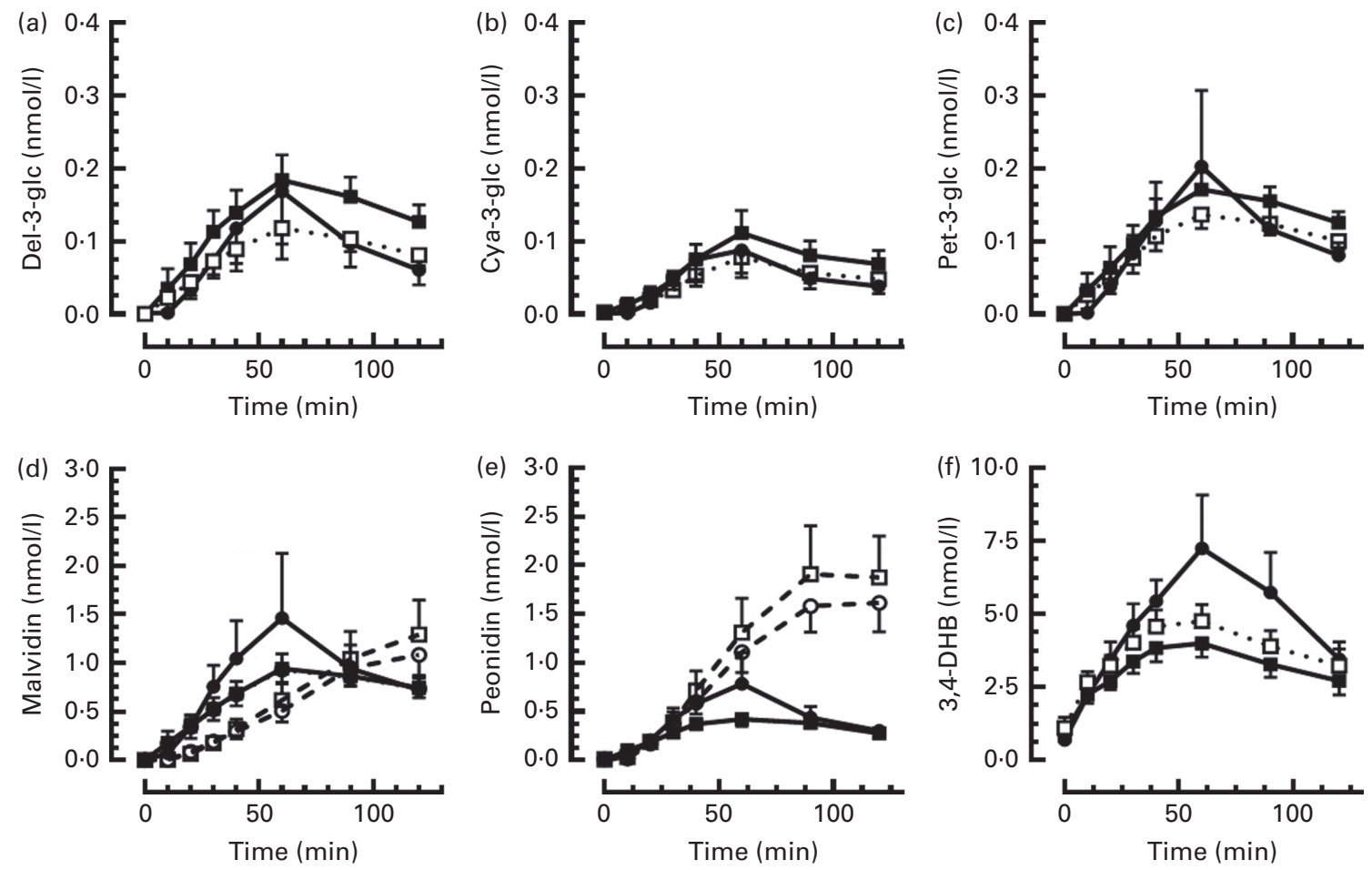

Fig. 4. Plasma concentrations of anthocyanin and 3,4-dihydroxybenzoic acid (3,4-DHB) from juice ( - -) or smoothie (non-adjusted ( --$)$ and adjusted (- $\square$-)) and their glucuronides (juice (-o-) and smoothie (- $\square-)$ ) in vivo. Values are means ( $n 10$ in duplicates), with their standard errors represented by vertical bars for (a) delphinidin-3-glucoside (Del-3-glc), (b) cyanidin-3-glucoside (Cya-3-glc), (c) petunidin-3-glucoside (Pet-3-glc), (d) malvidin-3-glucoside/glucuronide, (e) peonidin3-glucoside/glucuronide as well as (f) 3,4-DHB

ACN from juice and smoothie. This was shown for both, non-adjusted and dose-adjusted Mal-3-glc and Peo-3-glc values. The pharmacokinetic profiles of glucuronides were different compared with those of the glucosides. Malvidin3-glucuronide (Mal-3-glucuro) had a $C_{\max }$ of $1 \cdot 1$ (SEm $0 \cdot 2$ ) and $1.3(\operatorname{sem} 0.3) \mathrm{nmol} / \mathrm{l}$ for the juice and smoothie, a $t_{\max }$ of 114 (SEM 4) and 117 (SEM 3) min and an AUC of 64 (SEM 12) and 73 (sem 20), respectively. Peonidin-3-glucuronide (Peo3-glucuro) had a higher $C_{\max }$ of 1.7 (sem 0.3 ) and $2 \cdot 1$ $(\operatorname{sem} 0.5) \mathrm{nmol} / \mathrm{l}$ for the juice and smoothie, a lower $t_{\max }$ of
105 (SEM 5) and 108 (SEM 5) min and a higher AUC of 114 (SEM 20) and $135(\operatorname{sem~34)~} \mathrm{nmol} \times \mathrm{min} / \mathrm{l}$, respectively. It was not possible to determine the $t_{1 / 2}$ because the levels had not yet declined to baseline after reaching $C_{\max }$. This is most evident considering the pharmacokinetic profiles of glucuronides. In contrast to the two major $\mathrm{ACN}$, malvidin and peonidin, the minor ACN were only detected as glucosides with concentrations near the detection limit. Again, no significant differences were observed concerning the bioavailability of ACN from juice and smoothie (Table 2).

Table 2. Pharmacokinetic parameters of anthocyanins and phenolic acid in plasma (Mean values with their standard errors, $n 10$ in duplicate)

\begin{tabular}{|c|c|c|c|c|c|c|c|c|c|c|c|c|c|c|c|}
\hline & \multicolumn{4}{|c|}{$C_{\max }(\mathrm{nmol} / \mathrm{l})$} & \multicolumn{4}{|c|}{$t_{\max }(\min )$} & \multicolumn{4}{|c|}{$\mathrm{AUC}(\mathrm{nmol} \times \mathrm{min} / \mathrm{l})$} & \multicolumn{3}{|c|}{$A \cup C_{\text {adj }}(\mathrm{nmol} \times \mathrm{min} / \mathrm{l})$} \\
\hline & \multicolumn{2}{|c|}{ Juice } & \multicolumn{2}{|c|}{ Smoothie } & \multicolumn{2}{|c|}{ Juice } & \multicolumn{2}{|c|}{ Smoothie } & \multicolumn{2}{|c|}{ Juice } & \multicolumn{2}{|c|}{ Smoothie } & \multicolumn{2}{|c|}{ Smoothie } & \multirow{2}{*}{$\begin{array}{c}\text { Relative BV } \\
\text { Smoothie (\%) }\end{array}$} \\
\hline & Mean & SEM & Mean & SEM & Mean & SEM & Mean & SEM & Mean & SEM & Mean & SEM & Mean & SEM & \\
\hline Del-3-glc & 0.18 & 0.09 & 0.19 & 0.03 & 58 & 5 & 67 & 8 & 10 & 4 & 15 & 2 & 10 & 2 & $91 \cdot 7$ \\
\hline Cya-3-glc & 0.10 & 0.04 & 0.12 & 0.03 & 55 & 7 & 68 & 8 & $6 \cdot 0$ & $2 \cdot 1$ & $8 \cdot 2$ & $2 \cdot 1$ & $5 \cdot 8$ & 1.5 & $96 \cdot 5$ \\
\hline Pet-3-glc & 0.21 & 0.10 & 0.19 & 0.03 & 69 & 5 & 71 & 10 & 12 & 5 & 14 & $2 \cdot 2$ & 11 & 2 & 91.6 \\
\hline Mal-3-glc & 1.5 & 0.6 & $1 \cdot 0$ & 0.1 & 68 & 8 & 75 & 8 & 103 & 34 & 81 & 11 & 82 & 11 & $80 \cdot 0$ \\
\hline Peo-3-glc & $0 \cdot 80$ & 0.32 & 0.46 & 0.07 & 55 & 3 & 69 & 6 & 52 & 16 & 37 & 5 & 37 & 5 & $70 \cdot 9$ \\
\hline Mal-3-glucuro & 1.1 & 0.2 & 1.3 & 0.3 & 114 & 4 & 117 & 3 & 64 & 12 & 73 & 20 & & & \\
\hline Peo-3-glucuro & 1.7 & $0 . \overline{3}$ & $2 \cdot 1$ & 0.5 & 105 & 5 & 108 & 5 & 114 & 20 & 135 & 34 & & & \\
\hline 3,4-DHB & $7 \cdot 6$ & 1.8 & 4.5 & 0.5 & 57 & 5 & 63 & 8 & 568 & 79 & $285^{*}$ & 38 & $314^{*}$ & 47 & $79 \cdot 2^{*}$ \\
\hline
\end{tabular}

$C_{\max }$, maximal concentration; $t_{\max }$, time to maximal concentration; AUC ${ }_{a d j}$, AUC adjusted to ingested dose; BV, bioavailability; Del-3-glc, delphinidin-3-glucoside; Cya-3-glc, cyanidin-3-glucoside; Pet-3-glc, petunidin-3-glucoside; Mal-3-glc, malvidin-3-glucoside; Peo-3-glc, peonidin-3-glucoside; Mal-3-glucuro, malvidin-3-glucuronides; Peo-3-glucuro, peonidin-3-glucuronides; 3,4-DHB, 3,4-dihydroxybenzoic acid.

* Mean value was significantly different $(P<0.05)$. 
In contrast to the ACN and their metabolites, there was a significant difference in the bioavailability of the PA 3,4-DHB from juice and smoothie. The pharmacokinetic profiles (Fig. 4(f)) and parameters (Table 2) showed that 3,4-DHB was better bioavailable from juice than from smoothie. $C_{\max }$, $t_{\max }$ and AUC values for juice were 7.6 (SEm 1.8) nmol/1, 57 (SEM 5) $\mathrm{min}$ and $568(\mathrm{SEM} 79) \mathrm{nmol} \times \mathrm{min} / \mathrm{l}$, respectively. In contrast, $C_{\max }, t_{\max }$ and AUC values for smoothie were 4.5 (SEM 0.5) nmol/l, 63 (SEM 8) $\mathrm{min}$ and 285 (SEM 38) nmol $\times$ $\mathrm{min} / 1$, respectively, i.e. after adjustment to the dose in smoothie, the AUC value was $314(\operatorname{sem} 47) \mathrm{nmol} \times \mathrm{min} / \mathrm{l}$.

All ACN and their metabolites as well as the PA identified in the plasma were also detected in urine after the ingestion of juice or smoothie. Urinary samples were collected at intervals of $0-3,3-6,6-9,9-12,12-15$ and $15-24 \mathrm{~h}$, and the excretion of both glucosides and glucuronides peaked within the first two time intervals $(0-3$ and $3-6 \mathrm{~h})$ and were detectable until $12 \mathrm{~h}$ after ACN intake. The cumulative excretion curves of the ACN and their glucuronidated metabolites are shown in Fig. 5.

The main urinary ACN in both juice and smoothie were Mal-3-glc with 2.46 (SEM 0.44 ) and 2.64 (SEM 0.44$) \mathrm{nmol} / \mathrm{l}$ and Peo-3-glc with 2.65 (SEM 0.57) and 2.22 (SEM 0.40) nmol/1 being excreted until $24 \mathrm{~h}$ after ACN intake (Table 3). Substantial amounts of the minor ACN Del-3-glc, Cya-3-glc and Pet-3-glc were also detected, although in lower concentrations and without significant differences between juice and smoothie (0.27 (SEM 0.08) v. 0.34 (SEM 0.07), 0.56 (SEM 0.19) v. $0.36(\operatorname{sem} 0.07)$ and $0.39($ SEM 0.08) v. $0.46($ SEM 0.08) $\mathrm{nmol} / 1$, respectively). Glucuronidated metabolites of malvidin and peonidin occurred in urine with Peo-3-glucuro revealing the highest concentrations (3.61 (SEM 0.52) and 4.01 (SEM $0.53) \mathrm{nmol} / \mathrm{l}$ after juice and smoothie intake, respectively) compared with Mal-3-glucuro with 1.2 (SEM 0.2) and 1.3 (SEM $0 \cdot 2) \mathrm{nmol} / \mathrm{l}$ after juice and smoothie intake, respectively. In contrast to plasma, urinary excretion of 3,4-DHB reached high values after juice and smoothie ingestion, but without significant difference (23 (SEM 20) v. 10 (SEM 8) nmol/l) after baseline correction.

\section{Discussion}

Since ACN from juice or smoothie have a great beneficial potential concerning health and possibly served as an additional portion to increase the FV intake, it is of great importance to investigate their bioavailability in humans. Thus, the present study investigated the bioavailability of phenolic compounds from an ACN-rich juice and smoothie in vivo as well as the cellular uptake from a corresponding extract in vitro. This involved the assessment of (1) the stability, degradation and transport rates of ACN using Caco-2 cells as absorptive intestinal epithelial cells, (2) the stability, the degradation and transport rates of $\mathrm{PA} 3,4-\mathrm{DHB}$ in vitro, (3) the identification and quantification of $\mathrm{ACN}$ and their metabolites in plasma and urine as well as (4) the quantification of 3,4-DHB in (a)

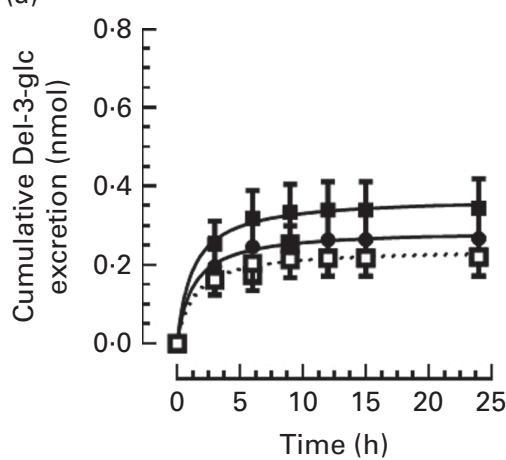

(d)

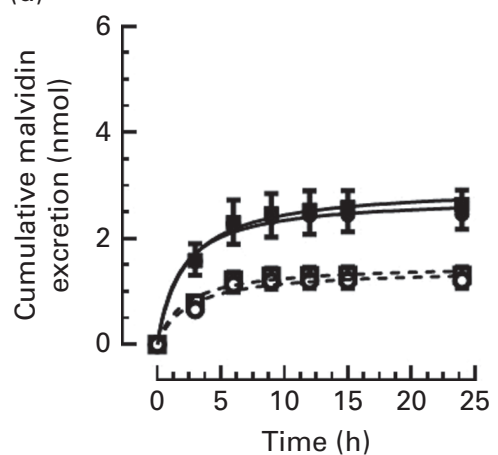

(b)

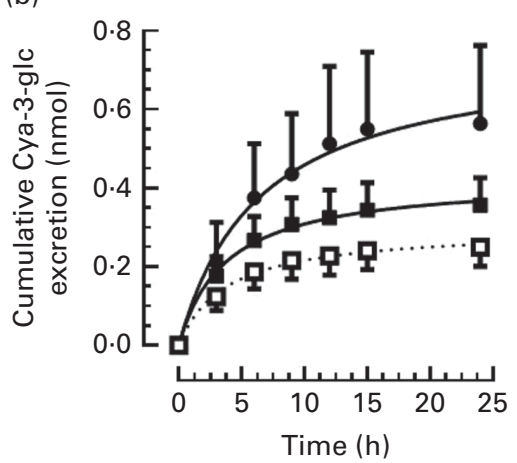

(e)

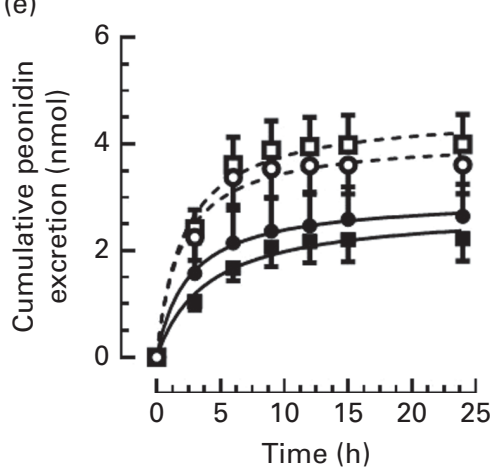

(c)

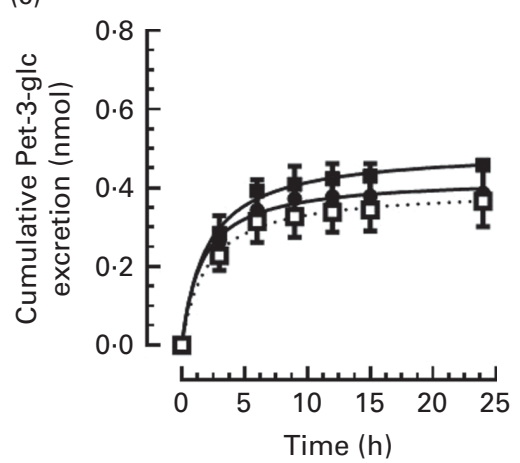

(f)

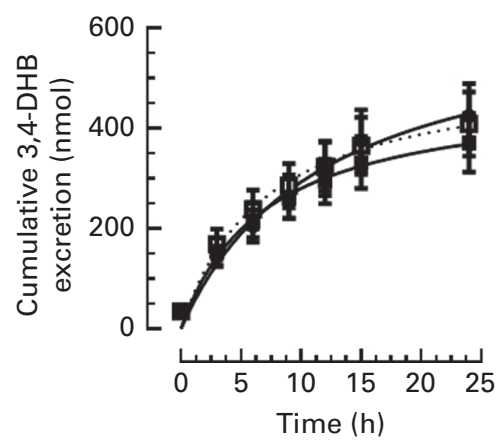

Fig. 5. Cumulative urinary concentrations of anthocyanin and 3,4-dihydroxybenzoic acid (3,4-DHB) from juice (-๑) or smoothie (non-adjusted (- -) and adjusted $(-\square)$ ) and their glucuronides (juice (-०.) and smoothie (- $\square)$ ) in vivo. Values are means ( $n 10$ in duplicate), with their standard errors represented by vertical bars for (a) delphinidin-3-glucoside (Del-3-glc), (b) cyanidin-3-glucoside (Cya-3-glc), (c) petunidin-3-glucoside (Pet-3-glc), (d) malvidin-3-glucoside/glucuronide and (e) peonidin-3-glucoside/glucuronide. 
Table 3. Pharmacokinetic parameters of anthocyanins and phenolic acid in urine

(Mean values with their standard errors, $n 10$ in duplicate)

\begin{tabular}{|c|c|c|c|c|c|c|}
\hline \multirow[b]{3}{*}{ Parameters } & \multicolumn{4}{|c|}{ Cumulative $C_{\max }(\mathrm{nmol} / \mathrm{l})$} & \multirow{2}{*}{\multicolumn{2}{|c|}{$\begin{array}{c}\text { Cumulative } \\
\begin{array}{c}C_{\text {max adj }} \\
(\mathrm{nmol} / \mathrm{l})\end{array} \\
\text { Smoothie }\end{array}$}} \\
\hline & \multicolumn{2}{|c|}{ Juice } & \multicolumn{2}{|c|}{ Smoothie } & & \\
\hline & Mean & SEM & Mean & SEM & Mean & SEM \\
\hline Del-3-glc & 0.27 & 0.08 & 0.34 & 0.07 & 0.22 & 0.05 \\
\hline Cya-3-glc & 0.56 & 0.19 & 0.36 & 0.07 & 0.25 & 0.05 \\
\hline Pet-3-glc & 0.39 & 0.08 & 0.46 & 0.08 & 0.37 & 0.06 \\
\hline Mal-3-glc & $2 \cdot 4$ & 0.4 & $2 \cdot 6$ & 0.4 & $2 \cdot 6$ & 0.4 \\
\hline Peo-3-glc & $2 \cdot 6$ & 0.6 & $2 \cdot 2$ & 0.4 & $2 \cdot 2$ & 0.4 \\
\hline Mal-3-glucuro & 1.2 & 0.2 & 1.3 & 0.2 & 1.3 & 0.2 \\
\hline Peo-3-glucuro & 3.6 & 0.5 & $4 \cdot 0$ & 0.5 & 4.0 & 0.5 \\
\hline 3,4-DHB & 23 & 20 & 10 & 8 & 12 & 10 \\
\hline
\end{tabular}

$C_{\max }$, maximal concentration; $C_{\max }$ adj, maximal concentration adjusted to ingested dose; Del-3-glc, delphinidin-3-glucoside; Cya-3-glc, cyanidin-3-glucoside; Pet-3-glc, petunidin-3-glucoside; Mal-3-glc, malvidin-3-glucoside; Peo-3-glc, peonidin-3-glucoside; Mal-3-glucuro, malvidin-3-glucuronides; Peo-3-glucuro, peonidin-3-glucuronides; 3,4-DHB, 3,4-dihydroxybenzoic acid.

plasma and urine in vivo after ingestion of an ACN-rich juice or smoothie.

1. In vitro, we evaluated the transport of ACN from a grape/ blueberry extract in Caco-2 cells using a two-compartment model. We showed that the five main glycones Mal-3-glc, Peo-3-glc, Pet-3-glc, Del-3-glc and Cya-3-glc in the extract were transported across the Caco- 2 cell monolayers in their intact glycone form, although with low transport efficiency, which was in accordance with previous in vitro studies. While Steinert et al. ${ }^{(34)}$ demonstrated the disappearance of black currant ACN from the apical compartment, but no occurrence in the basal compartment, Yi et al. ${ }^{(35)}$ and Liu et al. ${ }^{(22)}$ showed that ACN from blueberry extract were detected in the basal compartment. Similar to our data, transport efficiencies were dependent on the individual ACN with the highest recovery rates found in the basal compartments of Peo-3-glc $>$ Cya-3-glc $>$ Mal-3-glc $>$ Pet-3-glc $>$ Del-3-glc We observed no correlation between structure and recovery rates, although facilitated uptake into cells was shown for ACN with chemical structures exerting greater hydrophobicity (e.g. malvidin and peonidin) than those with higher numbers of hydroxyl groups (e.g. delphinidin) ${ }^{(35)}$ In our study, we found different recovery rates with Peo3-glc and Pet-3-glc despite their similar structure (four hydroxyl and one methyl group). In contrast, Cya-3-glc, for example, has four hydroxyl groups and no methyl group; however, the recovery rates were higher than that of Pet-3-glc. The fast disappearance of ACN in the apical solution and relatively low concentrations in the basal compartment could also be due to chemically induced degradation that may occur not only under in vitro conditions but also in vivo. Under acidic ( $\mathrm{pH} 2 \cdot 0)$ and neutral $(\mathrm{pH} 7 \cdot 4)$ conditions in the absence of Caco-2 cells, ACN were observed to be stable at $\mathrm{pH} 2 \cdot 0$, but were degraded at $\mathrm{pH} 7 \cdot 4$ with more than a $40 \%$ loss within the first 30 min for Mal-3-glc. Since the decreasing ACN rates at $\mathrm{pH} 7 \cdot 4$ were lower in the degradation studies (without cells) than in the incubation studies (with cells), it is assumed that a part of the apical disappearance of ACN was a result of their uptake across the cells. Due to these observations and the occurrence of $\mathrm{ACN}$ in the basal compartment, it was speculated that ACN were transported by active transport systems, which has been reported for other flavonoids ${ }^{(36-39)}$. Recently, Manzano \& Williamson ${ }^{(40)}$ demonstrated that ACN such as pelargonidin-3-glucoside inhibited glucose uptake. Using Na-containing (GLUT sodium-glucose co-transporter 1 (SGLT1) and GLUT2, both active) and Na-free (only GLUT2, active) conditions, they showed that the inhibition of GLUT2 was greater than that of SGLT1. They further observed that PPT (polyphenols, PA and tannins) inhibited the glucose transport from the intestinal lumen into cells and the GLUT2-facilitated exit to the basolateral side. Thus, although the chemically induced degradation seemed to be substantial, ACN could be transported across the epithelial monolayer. It should be noted that no transitional increase in the concentration of their aglycones was observed in the present study because of the possible hydrolysis of the glucose unit, probably due to the rapid turnover into other degradation products.

2. Because of the low bioavailability, it is speculated that $\mathrm{ACN}$ are likely to be degraded either spontaneously or enzymatically into phenolic degradation products, which are then further metabolised ${ }^{(41-43)}$. However, it is difficult to discriminate between PA deriving from the product or from intestinal and microbial degradation processes. 3,4-DHB, for example, which was present in high amounts in our test juice, smoothie and extract could have also been a degradation product of Cya-3glc $^{(44-46)}$. Hidalgo et al. ${ }^{(47)}$ detected degradation products of ACN in low amounts after incubation with human gut microbiota for $4-6 \mathrm{~h}$. Thus, under our conditions, we would exclude that the occurrence of PA in the basal compartment was a result of the cleavage of $\mathrm{ACN}$, but revealed 3,4-DHB transport from the extract, since PA transport takes place in the upper part of the gastrointestinal tract with a low microbe density in healthy subjects. Recently, Wang et al. ${ }^{(48)}$ demonstrated for the first time the simultaneous quantification of six PA after their oral administration to rats in plasma. They showed that 3,4-DHB was rapidly absorbed reaching $C_{\max }$ in less than $0.37 \mathrm{~h}$ with $t_{1 / 2}$ values of $5.75 \mathrm{~h}$ and mean $\mathrm{AUC}_{0 \rightarrow \infty}$ values of $789.7 \mathrm{ng} \times \mathrm{min} / \mathrm{ml}$. This indicates that 3,4-DHB was rapidly absorbed without further fermentation by the gut microbiota and reached high amounts in plasma. In accordance with these observations, we have shown in our in vitro study that $\mathrm{ACN}$ and the PA 3,4-DHB can be transported in their intact forms, albeit in low concentrations in comparison to their initial apical concentrations.

3. Existing literature on the bioavailability of $\mathrm{ACN}$ in vivo considering the effect of food matrix are scarce. If smoothie intake is an alternative to increase FV intake, 
we, therefore, compared the bioavailability of ACN from beverages with two different food matrices as ACN-rich juice and smoothie. The ACN profiles of the two beverages were nearly similar. In the case of the two main $\mathrm{ACN}, \mathrm{Mal}-3$-glc and Peo-3-glc, there was no difference between their content in juice and smoothie; however, Mal-3-glc concentration was higher than the Peo-3-glc concentration. After drinking 0.33 litres of juice or smoothie the AUC for Mal-3glc obtained from plasma were 103 and $81 \mathrm{nmol} \times \mathrm{min} / 1$ and $C_{\max }$ values were 1.54 and $1.0 \mathrm{nmol} / 1$, respectively. Furthermore, the $t_{\max }$ value of Mal-3-glc after juice intake was lower than that after smoothie ingestion, indicating that Mal-3-glc from juice was better available ( $80 \%$ relative bioavailability); however, because of the high individual variation, effects were not significant. Urinary excretion of Mal-3-glc was similar with 2.56 and $2.65 \mathrm{nmol} / 1$ for intact Mal-3-glc from juice and smoothie, respectively. Peo-3-glc seemed to be better available from juice than from smoothie indicated by the relative bioavailability of Peo-3-glc from smoothie with $71 \%$. In order to compare the bioavailability of the ACN from juice and smoothie, plasma concentrations were corrected for the actual amounts of $\mathrm{ACN}$ ingested via juice and smoothie. We could show that after adjustment of the pharmacokinetic parameters to the ACN dosages from juice and smoothie, the bioavailability of Del-3-glc, Cya-3-glc and Pet-3-glc was found to be about 5-10\% lower after smoothie intake than after juice ingestion. Similar to Stalmach et al. ${ }^{(49)}$ who analysed ACN and their metabolites in plasma and urine after ingestion of Concord grape juice $(350 \mathrm{ml}$, $528 \mu \mathrm{mol}$ total phenolic compounds), we showed detectable quantities of glucuronides of Mal-3-glc and Peo-3-glc in plasma and urine, whereas no glucuronides were found for the minor ACN Del-3-glc, Pet-3-glc and Cya3-glc. The delayed occurrence of Mal-3-glucuro and Peo-3-glucuro in plasma may be a consequence of post-absorption phase II metabolism converting the parental ACN structures to glucuronides in the gut and liver. Furthermore, although plasma malvidin and peonidin levels were in a similar range, Peo-3-glucuro was detected in higher amounts not only in plasma but also in urine compared with Mal-3-glucuro. In contrast to Stalmach et al. ${ }^{(49)}$ who speculated that the low petunidin: delphinidin ratio in plasma and an enhanced ratio in urine were a consequence of $5^{\prime}$-methylation of delphinidin in the kidney, our results indicate that the Peo-3-glc turnover in the circulatory system is faster than that of its malvidin counterparts as being reflected in increased plasma $C_{\max }$ levels. Alternatively, Cya-3-glc may have been methylated to Peo-3-glc and has been found as Peo-3-glucuro in plasma and urine after liver glucuronidation ${ }^{(50,51)}$. Although phase II glucuronidation takes place at any free hydroxyl position in the gut or liver, malvidin and peonidin glucuronides were identified as monoglucuronides.

4. In addition, structural differences of ACN interference factors such as food matrices or processing are important determinants of ACN bioavailability ${ }^{(52,53)}$. Del Bo et al. ${ }^{(54)}$ investigated the bioavailability of two different blueberry purees in healthy volunteers and showed that ACN absorption was higher from blanched berries than from a raw berry preparation. In a further study, investigating apple polyphenols, Hagl et al. ${ }^{(27)}$ have shown that substantially more polyphenols from apple smoothie reached the lower colon compared with those from apple juice. They speculated that absorption efficiencies were reduced after smoothie consumption because they contain more matrix components than juice. Hence, these components probably bind more polyphenols and thus reduce the bioavailability of the substances in the small intestine. In addition, the considerably higher polyphenol content of the apple smoothie could have reduced their relative bioavailability.

5. Interestingly, in this context, Kahle et al. ${ }^{(55)}$ investigate PA absorption in ileostomy volunteers and indicate that the amounts of hydroxycinnamic acids absorbed or metabolised are smaller after consumption of apple smoothie than after consumption of apple juice. Although in the present study no hydroxycinnamic acids were detected in the beverages or plasma, we have shown for 3,4DHB that the bioavailability of this PA was significantly lower after smoothie than after juice consumption. Plasma AUC values were 568 (SEM 79) after juice and $285($ sem 38$) \mathrm{nmol} \times \mathrm{min} / \mathrm{l}$ after smoothie consumption, respectively $314(\mathrm{SEM} 47) \mathrm{nmol} \times \mathrm{min} / \mathrm{l}$ after adjustment $\left(\right.$ AUC $\left._{\text {adj }}\right)$, with a relative bioavailability of $79 \%$ $(P<0.036)$. As 3,4-DHB could also be a degradation product of Cya-3-glc, it cannot be distinguished between 3,4-DHB deriving from the beverage and 3,4-DHB as a degradation product. In contrast, $t_{\max }$ values of the 3,4-DHB were in a similar range as Cya-3-glc, indicating that observed plasma levels were not a result of degradation processes in the colon.

In conclusion, the present study demonstrated that, despite their instability at neutral physiological $\mathrm{pH}$ and intense colonic metabolism, ACN from a berry extract were found at the serosal side in their native forms in vitro and their native and glucuronated forms in vivo after ingestion of the corresponding ACN-rich juice or smoothie. The liver seems to be the major site of post-absorptive ACN metabolism resulting in ACN monoglucuronides of the main ACN malvidin and peonidin. However, both in vitro and in vivo studies indicated that ACN absorption was below $0 \cdot 1 \%$ of the initial doses, indicating their intense degradation and fermentation. In contrast to ACN and their glucuronides, we detected a food-matrix effect for the hydroxybenzoic acid 3,4-DHB, resulting in a significant lower bioavailability after smoothie compared with juice consumption. We previously reported that both beverages improved antioxidative parameters such as antioxidative enzyme activities or plasma antioxidative capacity in a human intervention study. Nevertheless, it remains to be discussed whether smoothies and juice could be recommended as a substitute to improve FV intake, particularly for children. Comparable studies investigating the bioavailability of 
berry-derived ACN are scarce as well as the evidence of their health-promoting effects. This is a limitation of the present study, and should be investigated in further trials. Despite the acceptance of these beverages in adolescents and children, this source of health-promoting ACN has relatively high sugar content, which needs to be considered in the context of the growing problem of exceeding energy intake.

\section{Acknowledgements}

The authors thank Cordula Becker, Nadine Metz, Sina Streichert, Nicole Tscherney, Bianca Walber and Marcel Zoremba for their excellent technical assistance in performing blood sampling, cell culture and analytical preparations of plasma and urine samples, and also thank all volunteers for participating in the study.

The present study was funded by the Federal Ministry of Education and Research (BMBF) as part of the research consortium ANTHONIA (coordinator: C. K., grant no. 0315379A); the analysis was also supported by the German Research Foundation (B. S., DFG grant no. Sp314/12-1). The BMBF and DFG had no role in the study design, data collection and analysis; in the decision to publish or in the writing of this article.

The authors' contributions are as follows: S. K., S. R. and C. K. were the principal investigators of the present study, were responsible for the research questions, and involved in the study design and in the writing of the manuscript; H. A. contributed to the in vitro study, and C. B. contributed to the randomisation strategy and the handling of human samples; B. F. produced ACN-rich and ACN-depleted beverages and extracts, and measured $\mathrm{ACN}$ content; S. D., F. U., A. R. and B. S. were responsible for quantification and identification of ACN/ACN metabolites and PA in plasma, urine and cell culture samples.

The authors declare that there are no conflicts of interest.

\section{References}

1. Liu S, Lee IM, Ajani U, et al. (2001) Intake of vegetables rich in carotenoids and risk of coronary heart disease in men: the physicians' health study. Int J Epidemiol 30, 130-135.

2. He FJ, Nowson CA \& MacGregor GA (2006) Fruit and vegetable consumption and stroke: meta-analysis of cohort studies. Lancet 367, 320-326.

3. Gaziano JM, Manson JE, Branch LG, et al. (1995) A prospective study of consumption of carotenoids in fruits and vegetables and decreased cardiovascular mortality in the elderly. Ann Epidemiol 5, 255-260.

4. Nöthlings U, Schulze MB, Weikert C, et al. (2008) Intake of vegetables, legumes, and fruit, and risk for all-cause, cardiovascular, and cancer mortality in a European diabetic population. J Nutr 138, 775-781.

5. Evans CE, Christian MS, Cleghorn CL, et al. (2012) Systematic review and meta-analysis of school-based interventions to improve daily fruit and vegetable intake in children aged 5 to 12 y. Am J Clin Nutr 96, 889-901.

6. Christian MS, Evans CE, Hancock N, et al. (2013) Family meals can help children reach their 5 a day: a cross-sectional survey of children's dietary intake from London primary schools. J Epidemiol Community Health 67, 332-338.

7. Glen KE, Thomas HM, Loebach JE, et al. (2013) Fruit and vegetable consumption among children in a socioeconomically disadvantaged neighbourhood. Can J Diet Pract Res 74, 114-118.

8. Coppinger T, Jeanes Y, Mitchell M, et al. (2013) Beverage consumption and BMI of British schoolchildren aged 9-13 years. Public Health Nutr 16, 1244-1249.

9. Maatta K, Kamal-Eldin A \& Törrönen R (2001) Phenolic compounds in berries of black, red, green, and white currants (Ribes sp.). Antioxid Redox Signal 3, 981-993.

10. Mikkonen TP, Määttä KR, Hukkanen AT, et al. (2001) Flavonol content varies among black currant cultivars. J Agric Food Chem 49, 3274-3277.

11. Müller D, Schantz M \& Richling E (2012) High performance liquid chromatography analysis of anthocyanins in bilberries (Vaccinium myrtillus L.), blueberries (Vaccinium corymbosum L.), and corresponding juices. J Food Sci 77, 340-345.

12. Hertog MG, Hollman PC, Katan MB, et al. (1993) Intake of potentially anticarcinogenic flavonoids and their determinants in adults in The Netherlands. Nutr Cancer 20, 21-29.

13. Zamora-Ros R, Knaze V, Luján-Barroso L, et al. (2011) Estimation of the intake of anthocyanidins and their food sources in the European Prospective Investigation into Cancer and Nutrition (EPIC) study. Br J Nutr 106, 1090-1099.

14. Fang J (2014) Some anthocyanins could be efficiently absorbed across the gastrointestinal mucosa: extensive presystemic metabolism reduces apparent bioavailability. J Agric Food Chem 62, 3904-3911.

15. Vitaglione P, Donnarumma G, Napolitano A, et al. (2007) Protocatechuic acid is the major human metabolite of cyanidin-glucosides. J Nutr 137, 2043-2048.

16. Miyazawa T, Nakagawa K, Kudo M, et al. (1999) Direct intestinal absorption of red fruit anthocyanins, cyanidin3-glucoside and cyanidin-3,5-diglucoside, into rats and humans. J Agric Food Chem 47, 1083-1091.

17. Felgines C, Talavera S, Gonthier MP, et al. (2003) Strawberry anthocyanins are recovered in urine as glucuro- and sulfoconjugates in humans. J Nutr 133, 1296-1301.

18. Mullen W, Edwards CA, Serafini M, et al. (2008) Bioavailability of pelargonidin-3-O-glucoside and its metabolites in humans following the ingestion of strawberries with and without cream. J Agric Food Chem 56, 713-719.

19. Borges G, Mullen W, Mullan A, et al. (2010) Bioavailability of multiple components following acute ingestion of a polyphenol-rich juice drink. Mol Nutr Food Res 54, 268-277.

20. Galvano F, La Fauci L, Vitaglione P, et al. (2007) Bioavailability, antioxidant and biological properties of the natural free-radical scavengers cyanidin and related glycosides. Ann Ist Super Sanita 43, 382-393.

21. Hribar U \& Ulrih NP (2014) The metabolism of anthocyanins. Curr Drug Metab 15, 3-13.

22. Liu Y, Zhang D, Wu Y, et al. (2014) Stability and absorption of anthocyanins from blueberries subjected to a simulated digestion process. Int J Food Sci Nutr 65, 440-448.

23. de Ferrars RM, Cassidy A, Curtis P, et al. (2014) Phenolic metabolites of anthocyanins following a dietary intervention study in post-menopausal women. Mol Nutr Food Res $\mathbf{5 8}$, 490-502.

24. Yang M, Koo SI, Song WO, et al. (2011) Food matrix affecting anthocyanin bioavailability: review. Curr Med Chem 18, 291-300.

25. Mertens-Talcott SU, Rios J, Jilma-Stohlawetz P, et al. (2008) Pharmacokinetics of anthocyanins and antioxidant effects 
after the consumption of anthocyanin-rich acai juice and pulp (Euterpe oleracea Mart.) in human healthy volunteers. J Agric Food Chem 56, 7796-7802.

26. Toydemir G, Boyacioglu D, Capanoglu E, et al. (2013) Investigating the transport dynamics of anthocyanins from unprocessed fruit and processed fruit juice from sour cherry (Prunus cerasus L.) across intestinal epithelial cells. J Agric Food Chem 61, 11434-11441.

27. Hagl S, Deusser H, Soyalan B, et al. (2011) Colonic availability of polyphenols and D-(-)-quinic acid after apple smoothie consumption. Mol Nutr Food Res 55, 368-377.

28. Nurmi T, Mursu J, Heinonen M, et al. (2009) Metabolism of berry anthocyanins to phenolic acids in humans. J Agric Food Chem 57, 2274-2281.

29. Fröhling B, Patz C, Dietrich H, et al. (2012) Anthocyanins, total phenolics and antioxidant capacities of commercial red grape juices, black currant and sour cherry nectars. Fruit Process 3, 100-104.

30. Spormann TM, Albert FW, Rath T, et al. (2008) Anthocyanin/ polyphenolic-rich fruit juice reduces oxidative cell damage in an intervention study with patients on hemodialysis. Cancer Epidemiol Biomarkers Prev 17, 3372-3380.

31. Kuntz S, Kunz C, Herrmann J, et al. (2014) Anthocyanins from fruit juice improve antioxidant status of healthy young female volunteers without affecting anti-inflammatory parameters: results from the randomized double-blind placebo controlled cross-over ANTHONIA study. Br J Nutr 112, 925-936.

32. Esselen M, Barth SW, Winkler S, et al. (2013) Anthocyanins suppress the cleavable complex formation by irinotecan and diminish its DNA-strand-breaking activity in the colon of Wistar rats. Carcinogenesis 34, 835-840.

33. Urpi-Sarda M, Zamora-Ros $\mathrm{R}$, Lamuela-Raventos $\mathrm{R}$, et al. (2007) HPLC-tandem mass spectrometric method to characterize resveratrol metabolism in humans. Clin Chem $\mathbf{5 3}$, 292-299.

34. Steinert RE, Ditscheid B, Netzel M, et al. (2008) Absorption of black currant anthocyanins by monolayers of human intestinal epithelial Caco-2 cells mounted in using type chambers. J Agric Food Chem 56, 4995-5001.

35. Yi W, Akoh CC, Fischer J, et al. (2006) Absorption of anthocyanins from blueberry extracts by caco-2 human intestinal cell monolayers. J Agric Food Chem 54, 5651-5658.

36. Kottra G \& Daniel H (2007) Flavonoid glycosides are not transported by the human $\mathrm{Na}^{+} /$glucose transporter when expressed in Xenopus laevis oocytes, but effectively inhibit electrogenic glucose uptake. J Pharmacol Exp Ther 322, 829-835.

37. Walgren RA, Karnaky KJ, Lindenmayer GE, et al. (2000) Efflux of dietary flavonoid quercetin $4^{\prime}$-beta-glucoside across human intestinal Caco-2 cell monolayers by apical multidrug resistance-associated protein-2. J Pharmacol Exp Ther 294, 830-836.

38. Gee JM, DuPont MS, Rhodes MJ, et al. (1998) Quercetin glucosides interact with the intestinal glucose transport pathway. Free Radic Biol Med 25, 19-25.

39. Walgren RA, Lin JT, Kinne RK, et al. (2000) Cellular uptake of dietary flavonoid quercetin 4'-beta-glucoside by sodiumdependent glucose transporter SGLT1. J Pharmacol Exp Ther 294, 837-843.
40. Manzano S \& Williamson G (2010) Polyphenols and phenolic acids from strawberry and apple decrease glucose uptake and transport by human intestinal Caco-2 cells. Mol Nutr Food Res 54, 1773-1780.

41. Woodward G, Kroon P, Cassidy A, et al. (2009) Anthocyanin stability and recovery: implications for the analysis of clinical and experimental samples. I Agric Food Chem 57, 5271-5278.

42. Williamson G \& Clifford M (2010) Colonic metabolites of berry polyphenols: the missing link to biological activity? Br J Nutr 104, 48-66.

43. González-Barrio R, Edwards C \& Crozier A (2011) Colonic catabolism of ellagitannins, ellagic acid, and raspberry anthocyanins: in vivo and in vitro studies. Drug Metab Dispos 39, 1680-1688.

44. Hanske L, Engst W, Loh G, et al. (2013) Contribution of gut bacteria to the metabolism of cyanidin 3-glucoside in human microbiota-associated rats. Br J Nutr 109, 1433-1441.

45. Aura AM, Martin-Lopez P, O'Leary KA, et al. (2005) In vitro metabolism of anthocyanins by human gut microflora. Eur J Nutr 44, 133-142.

46. Fleschhut J, Kratzer F, Rechkemmer G, et al. (2006) Stability and biotransformation of various dietary anthocyanins in vitro. Eur J Nutr 45, 7-18.

47. Hidalgo M, Oruna-Concha MJ, Kolida S, et al. (2012) Metabolism of anthocyanins by human gut microflora and their influence on gut bacterial growth. J Agric Food Chem 60, 3882-3890.

48. Wang XY, Ma XH, Li W, et al. (2013) Simultaneous determination of five phenolic components and paeoniflorin in rat plasma by liquid chromatography-tandem mass spectrometry and pharmacokinetic study after oral administration of Cerebralcare granule ${ }^{(\circledR)}$. J Pharm Biomed Anal 86, 82-91.

49. Stalmach A, Edwards CA, Wightman JD, et al. (2012) Gastrointestinal stability and bioavailability of (poly) phenolic compounds following ingestion of Concord grape juice by humans. Mol Nutr Food Res 56, 497-509.

50. Fernandes I, Azevedo J, Faria A, et al. (2009) Enzymatic hemisynthesis of metabolites and conjugates of anthocyanins. I Agric Food Chem 57, 735-745.

51. Marques F, de Freitas V \& Mateus N (2013) Antioxidant and antiproliferative properties of methylated metabolites of anthocyanins. Food Chem 141, 2923-2933.

52. Rodriguez-Mateos A, Pino-García RD, George TW, et al. (2014) Impact of processing on the bioavailability and vascular effects of blueberry (poly) phenols. Mol Nutr Food Res 58, 1952-1961.

53. Rodriguez-Mateos A, Cifuentes-Gomez T, George TW, et al. (2013) Impact of cooking, proving, and baking on the (poly)phenol content of wild blueberry. J Agric Food Chem 62, 3979-3986.

54. Del Bo' C, Riso P, Brambilla A, et al. (2012) Blanching improves anthocyanin absorption from highbush blueberry (Vaccinium corymbosum L.) purée in healthy human volunteers: a pilot study. J Agric Food Chem 60, 9298-9304.

55. Kahle K, Kraus M, Scheppach W, et al. (2005) Colonic availability of apple polyphenols - a study in ileostomy subjects. Mol Nutr Food Res 49, 1143-1150. 